\title{
Sun-Struck Scientist
}

\author{
Nicola Fox, who leads NASA's heliophysics division, explains what it \\ means to "touch the Sun."
}

By Michael Schirber

$\mathrm{N}$ icola Fox loved space as a kid and was fascinated by the workings of the world. When it came time to decide on a career path, her passion for space led her to study satellite engineering. But it quickly became clear that this focus wasn't the right one for her. "My professors kept telling me that I asked the wrong questions," she says. "I was more interested in why than how."

Fox switched to studying space plasma physics and eventually joined NASA. In 2010, she became the project scientist for the Parker Solar Probe, a mission to sample the Sun's atmosphere (see To Touch the Sun). That role has, in some sense, brought her career full circle, as she worked hand in hand with engineers to make the probe ready for its mission to "touch the Sun."

Just after the probe launched in August 2018, Fox became the director of the heliophysics division at NASA. Physics spoke to her about her interest in the Sun and whether she worries about the Parker Solar Probe as it spirals ever closer to that ball of fire.

All interviews are edited for brevity and clarity.

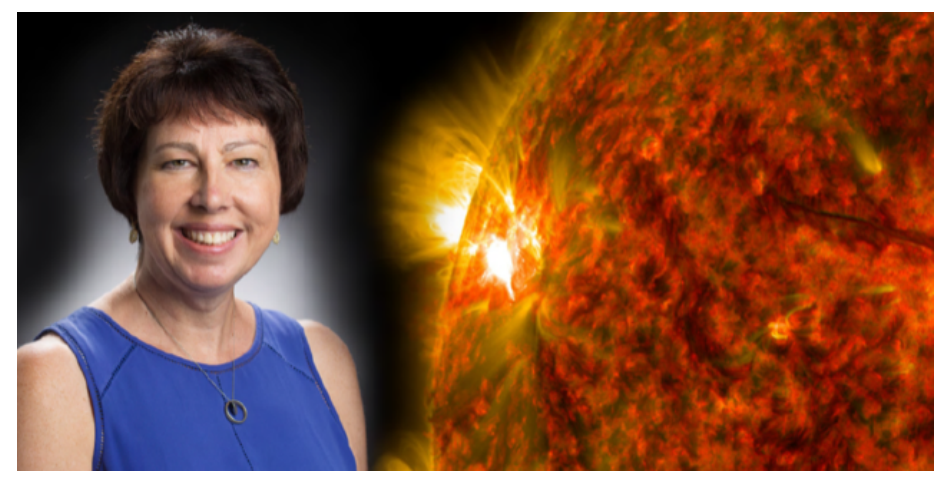

Credit: NASA

\section{How did you end up a solar scientist?}

I actually started out studying Earth's magnetosphere and the auroras. The first big project that I worked on at NASA was the Van Allen Probes mission that looked at the high-energy particles in Earth's radiation belts. The Sun and its solar wind are important for feeding energy into those belts, but it wasn't until I joined the Parker Solar Probe mission that I really got fascinated with the Sun itself.

\section{As the probe's project scientist, what was your main role?}

The number one responsibility of a project scientist is ensuring that the mission delivers the science. I worked with the engineering team that was designing the probe to meet the science objectives. We sometimes had to make compromises-relaxing a science requirement a little bit to address an engineering constraint. But in the end, we succeeded in meeting the scientific goals. I must say that I had a fantastic team that never stepped away from a challenge.

\section{On April 28 of this year, the probe came within 7.5 million miles (12 million kilometers) of the Sun's surface. For a few hours, it entered the Sun's magnetically dominated corona. Can you describe what that region is?}

It's a region of space where the magnetic field is very strong and still tied to the Sun. I've often described it as the place where the magic happens and where the mysteries occur. One mystery is why the Sun's atmosphere, or corona, is 300 times hotter than its visible surface-where the gas is sufficiently dense to emit lots of light. Another is how the solar wind becomes energized. That energizing occurs in the region of the corona that the probe has dipped into.

\section{So the probe is "touching the Sun," as some people}




\section{have said?}

The Sun has no solid surface; it's just a churning ball of gas. So really, you can't touch the Sun like you would touch the ground of a planet. But the probe touched the Sun's atmosphere. In particular, it sampled the part of the Sun's atmosphere that drives space weather, which can affect satellites and power grids on Earth.

\section{Is this moment akin to that of planting a flag on the moon?}

I actually think it is. The probe entered a place that scientists have a lot of unproven hypotheses about. We haven't been able to prove them because, until now, we could only observe this region of space from afar. It's kind of like having a photograph of a distant relative whom you've never met. You may know what they look like, but you don't know much about their character. It's the same with the Sun. To understand its atmosphere, we have to go there and sample its density, composition, and temperature.

\section{What information are you most excited about the probe sending back?}

I'm looking forward to data that will test the ideas of Gene Parker-for whom the probe is named. In the 1950s, Gene predicted the solar wind, but almost nobody believed him. Since then, he has made other predictions, such as the existence of nanoflares for heating the corona. If nanoflares occur, we expect that the Parker Solar Probe will measure a particular radio signature as it gets closer to the Sun. Gene was there for the probe's launch, and I think it would be great if the probe told us he was right all along about the Sun.

\section{Where is the probe now?}

It just completed its 10th "close" approach to the Sun and has 14 more to go. The last one will be in June 2025 and will bring the probe to within 3.8 million miles (6.1 million kilometers) of the visible surface.

\section{Are you worried that the probe might get damaged as it flies closer to the Sun?}

Of course. When you work so hard on a spacecraft, you become very attached to it. The probe is part of the team, and we know that "she" is going into a very unforgiving environment. A lot of planning was done ahead of time to make sure that the spacecraft could look after itself. But there are long stretches-five, six, seven days-when the probe is so close to the Sun that we can't receive messages from it. During that time, the team waits on tenterhooks for a "green" tone, which is the "okay" radio signal from the probe. I know what time that tone is supposed to come in, and I sit with my phone in my hand waiting for the text from the operations team. They know I'm worried, so when the signal arrives, they send me a little green heart. Then I know that the probe is okay.

Michael Schirber is a Corresponding Editor for Physics based in Lyon, France. 